\title{
Subsumer-First: Steering Symbolic Reachability Analysis
}

\author{
Andrey Rybalchenko ${ }^{1}$ and Rishabh Singh ${ }^{2}$ \\ 1 Max Planck Institute for Software Systems (MPI-SWS) \\ 2 Massachusetts Institue of Technology (MIT)
}

\begin{abstract}
Symbolic reachability analysis provides a basis for the verification of software systems by offering algorithmic support for the exploration of the program state space when searching for proofs or counterexamples. The choice of exploration strategy employed by the analysis has direct impact on its success, whereas the ability to find short counterexamples quickly and - as a complementary task - to efficiently perform the exhaustive state space traversal are of utmost importance for the majority of verification efforts. Existing exploration strategies can optimize only one of these objectives which leads to a sub-optimal reachability analysis, e.g., breadth-first search may sacrifice the exploration efficiency and chaotic iteration can miss minimal counterexamples. In this paper we present subsumer-first, a new approach for steering symbolic reachability analysis that targets both minimal counterexample discovery and efficiency of exhaustive exploration. Our approach leverages the result of fixpoint checks performed during symbolic reachability analysis to bias the exploration strategy towards its objectives, and does not require any additional computation. We demonstrate how the subsumer-first approach can be applied to improve efficiency of software verification tools based on predicate abstraction. Our experimental evaluation indicates the practical usefulness of the approach: we observe significant efficiency improvements (median value $40 \%$ ) on difficult verification benchmarks from the transportation domain.
\end{abstract}

\section{Introduction}

Model Checking [7] is a popular verification technology employed to verify both hardware and software systems $[1,5,14]$. State space exploration using symbolic techniques provides a basis for the verification of software systems. Explicit-state model checking which is commonly used to analyze software systems face the fundamental problem of state space explosion during this exploration. Scalability is one of the major challenges that model checking techniques face today which limits their applicability to verifying large systems. There are various efforts made to overcome this bottleneck. Techniques like abstract interpretation [8] try to abstract only relevant properties of the program to prove its correctness. Symbolic model checking [4] avoids explicit construction of the state space by performing symbolic fixpoint computation. Partial-order reduction [17] tries 
to explore only representative transitions and ignores other redundant transitions. But these techniques still suffer from having to explore a huge state space, which in turn is greatly dependent on the search strategy used for exploration. The exploration procedure used has a significant direct impact on the overall effectiveness of the verification efforts.

Directed model checking techniques $[10,11]$ try to direct the state space search to avoid the potential blowup faced by uninformed model checking techniques. Various heuristic strategies $[9,13,15]$ have been proposed for searching the state space efficiently. Saturating the strongly connected components first [3] is also proposed for efficient search. But all these searching techniques aim to optimize only one objective, e.g. the counterexample length or the efficient traversal of the state space. Heuristics directed towards quickly finding the error state may not scale very well in the absence of error states and vice versa.

In this paper we present "subsumer-first", a new approach for steering symbolic reachability analysis that targets both minimal counterexample discovery and efficiency of exhaustive exploration. The subsumer-first approach leverages the results of partial fixpoint checks - subsumption tests - performed during the symbolic state space exploration. The main idea is to schedule the successor computation of a symbolic state, say $s$, before the successor computation of all states that are reachable from the states subsumed $s$.

Subsumer-first has implicit bias towards the discovery of symbolic counterexamples that maximize the size of the symbolic state that reaches the error location. This aspect of the heuristic is useful for improving the effectiveness of the counterexample-guided abstraction refinement procedures, since it exposes the imprecision of the abstraction to a larger extent.

We present an application of the subsumer-first heuristic for improving the efficiency of abstraction-based software verification within the framework of CEGAR [6] based approaches. The empirical evaluation on industrial benchmarks of this search strategy implemented in ARMC [18], a predicate abstraction based model checker, presents its efficacy. The search strategy saves in the abstraction computation phase across the refinement iterations. We observe significant efficiency improvements (median of $40 \%$ reduction, 1.6 speedup) on difficult benchmarks from the transportation domain. The subsumer guided search on an average leads to a significant reduction in the number of abstraction entailment queries, total number of states explored and the total time taken. In some cases it is orders of magnitude faster than the breadth-first strategy successfully employed previously.

\section{Example}

In this section, we illustrate the subsumer-first approach on a simple example motivated by the symbolic reachability analysis.

We consider a set of symbolic program states that contains a distinguished start state. We assume that the set of symbolic states are partially ordered by the subset inclusion relation on the sets of concrete states denoted by the 


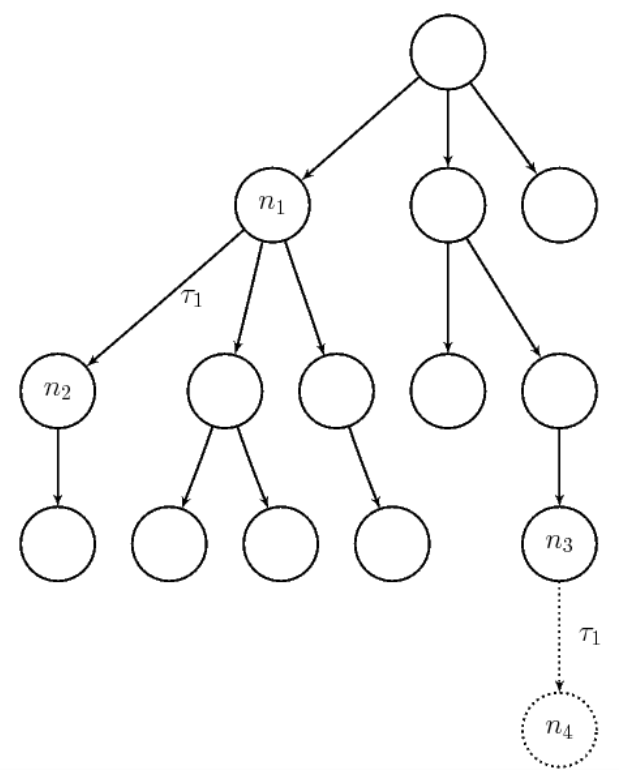

Fig. 1. Reachability tree fragment.

corresponding symbolic states. We say a symbolic state $n_{1}$ subsumes a symbolic state $n_{2}$ if the state $n_{1}$ is larger than $n_{2}$ with respect to the given partial order. Furthermore, we consider a finite collection of monotonic successor functions over the symbolic states which represent a transition relation. Our goal is to construct a tree whose nodes are symbolic states, whose root is the start state, and whose parent-child relation is determined by the given transition relation.

We consider a reachability tree fragment in Figure 1, and assume that it was constructed in the breadth-first manner. Let $n_{3}$ be the last node that was added to the tree, and furthermore assume that $n_{1}$ subsumes a node $n_{1}$. All the nodes that are reachable from node $n_{1}$ are also reachable from $n_{3}$. Now there are a few alternatives to resume the search from this point onwards.

One possible option is to continue the search in breadth-first manner, i.e. append node $n_{3}$ at the end of the queue of nodes to be expanded and continue. However, this approach can be sub-optimal since a significant amount of time may be spent in exploring states inside the subtree rooted at $n_{1}$ which are anyway going to be explored from state $n_{3}$ after some time. Such redundant computation should be avoided.

Another option is be to delete all the nodes in the subtree rooted at $n_{1}$ and resume the search in the breadth-first manner. Since all nodes reachable from $n_{1}$ are guaranteed to be reached from $n_{3}$, it is a sound step to perform. The problem with this approach is that we may lose on already computed states (nodes inside subtree rooted at $n_{1}$ ) and would need to recompute them again. If the number 
of nodes in the subtree to be deleted is large, we potentially lose significantly in the re-computation of all the pruned nodes.

Our subsumer-first approach keeps the tree intact instead of deleting the subtree rooted at node $n_{1}$. The node $n_{1}$ is pruned from the tree. The node $n_{3}$ is scheduled for expansion in the queue $\mathcal{Q}$ before the nodes in the subtree rooted at $n_{1}$ which are present in $\mathcal{Q}$. From the monotonicity of successor functions, the successor node of $n_{3}$ with respect to a function $\tau_{1}$, say $n_{4}$, is guaranteed to be at least as large as $n_{2}$. If the successor node $n_{4}$ is larger than $n_{2}$, then the same algorithm applies recursively: the node $n_{2}$ is pruned and $n_{4}$ is scheduled ahead of the nodes in the subtree rooted at node $n_{2}$. But if the successor node $n_{4}$ is equal to node $n_{2}$, then we prune the node $n_{4}$. Now we can reuse all of the previously computed subtree of $n_{2}$. So if at some point, the newly computed successor node becomes exactly equal to some previously computed node, we can stop searching the space further from that node and reuse the whole of the previously computed subtree. It is easy to envision the cases where significant savings can be achieved. The child and parent pointers need to be appropriately modified and maintained during the complete iteration.

Another important advantage of this approach is that since larger symbolic states (less constrained) get priority for the expansion first, we search for error states in a much larger state space. The counterexamples involving the larger symbolic states are given priority over the ones involving smaller states (more constrained). Therefore the counterexamples we get in the abstract-check-refine loop expose the imprecision of the abstraction to a larger extent. Another perspective on the obtained counterexample is to consider them as a logical combination of multiple counterexamples. Refining multiple counterexamples simultaneously has been shown empirically to perform well in practice [16], which is also confirmed by our experimental evaluation.

\section{Subsumer-first approach}

In this section, we present the subsumer-first approach in an abstract setting.

Let $(S, \leq)$ be a partially ordered set, $s_{0}$ be a distinguished element in $S$, and $R \subseteq S \times S$ be a binary relation over $S$ such that

$$
\forall s, s^{\prime}, t \in S \exists t^{\prime} \in S: s \leq t \wedge\left(s, s^{\prime}\right) \in R \rightarrow\left(t, t^{\prime}\right) \in R \wedge t \leq t^{\prime} .
$$

Intuitively, $S$ corresponds to a set of symbolic program states of a program and $R$ models the transition relation of the program, which is necessarily monotonic. Our goal is to compute the set of elements in $S$ that is reachable from $s_{0}$ via the binary relation $R$ in form of a reachability tree. We assume that the tree is computed using a worklist based algorithm, and leave the worklist scheduling as unspecified.

The subsumer-first approach amounts to the following exploration strategy. Let $s$ be an element to put on the worklist and $X$ be the set of all elements that are reachable from any element already appearing in the tree and subsumed 
by $s$, i.e.,

$X=\left\{s^{\prime \prime} \mid\right.$ exist already reached $s^{\prime}$ such that $s^{\prime} \leq s$ and $\left.\left(s^{\prime}, s^{\prime \prime}\right) \in R^{*}\right\}$.

Then, we put $s$ on the worklist at a position with higher priority than any element from the set $X$.

\section{Subsumer-first for predicate abstraction}

In this section we present an application of the subsumer-first approach for the symbolic reachability analysis using predicate abstraction, which is a prominent abstraction technique for software verification.

\subsection{Preliminaries}

This section provides basic definitions together with a brief description of predicate abstraction [2] and a refinement-based approach for proving safety properties (which can be skipped by experts in predicate abstraction).

Programs and Computations A program $P=\left(\Sigma, \mathcal{T}, s_{I}, S_{E}\right)$ consists of

$-\Sigma$ : a set of program states,

- $\mathcal{T}$ : a finite set of program transitions such that each transition $\tau \in \mathcal{T}$ is associated with a binary transition relation $\rho_{\tau} \subseteq \Sigma \times \Sigma$,

$-s_{I}:$ an initial state, $s_{I} \in \Sigma$,

- $S_{E}$ : a set of error states, $S_{E} \subseteq \Sigma$.

Our exposition does not assume any further state structure. Though, for the sake of concreteness we point out that usually a program state $s \in \Sigma$ is represented by a valuation of program variables, and program transitions $\mathcal{T}$ correspond to program statements as written in a programming language.

A program computation $\sigma=s_{1}, s_{2}, \ldots$ is a sequence of program states that starts at the initial state, i.e., $s_{1}=s_{I}$, and each pair of consecutive states $\left(s_{i}, s_{i+1}\right)$ is related by some program transition $\tau \in \mathcal{T}$, i.e., $\left(s_{i}, s_{i+1}\right) \in \rho_{\tau}$. A program state $s$ is reachable if it appears in some program computation. Let Reach be the set of all reachable states. The program $P$ is safe if no error state in $S_{E}$ is reachable in any computation, i.e., if $S_{E} \cap$ Reach $=\emptyset$.

A program path $\pi$ is a sequence of program transitions. We write $\pi \cdot \tau$ to denote an extension of a path $\pi$ by a transition $\tau$. A program path $\pi=\tau_{1}, \ldots, \tau_{n}$ is feasible if it induces a computation, i.e., if there is a sequence of states $s_{1}, \ldots, s_{n+1}$ such that $\left(s_{i}, s_{i+1}\right) \in \rho_{\tau_{i}}$ for each $1 \leq i \leq n$. 
Predicate Abstraction and Refinement We can verify program safety by computing the set of reachable program states and checking if it contains the error states. The set of reachable program states Reach can be constructed incrementally by iterating the "one-step" reachability operator post that maps a set of states $S \subseteq \Sigma$ into a set of immediate successors. Formally, for each transition $\tau \in \mathcal{T}$ we define

$$
\operatorname{post}(\tau, S)=\left\{s^{\prime} \in \Sigma \mid \exists s \in S:\left(s, s^{\prime}\right) \in \rho_{\tau}\right\},
$$

and then extend canonically to aggregate over all program transitions

$$
\operatorname{post}(S)=\bigcup_{\tau \in \mathcal{T}} \operatorname{post}(\tau, S) .
$$

Then, the set Reach of all reachable states consists of the states reachable from the initial state $s_{I}$ by any finite number of post-applications:

$$
\begin{aligned}
\text { Reach } & =\bigcup_{i \geq 0} \operatorname{post}^{i}\left(\left\{s_{I}\right\}\right) \\
& =\operatorname{lfp}\left(\text { post },\left\{s_{I}\right\}\right) .
\end{aligned}
$$

The set Reach of reachable states is generally not computable, since the number of iterations required to reach the fixpoint can be very large or infinite. For practical safety verification, we observe that any sufficiently precise overapproximation of Reach can be used to check program safety: if the error state is not present in the approximation then it is not reachable. Thus, by adjusting the precision of over-approximation we can achieve the desired practical effectiveness of the iterative reachability computation.

The framework of abstract interpretation formalizes the approximation-based approach by defining the effect of over-approximation using an abstraction function $\alpha$ as a basic building block [8]. The abstraction function $\alpha$ maps a set of program states to its over-approximation. Formally, we require $S \subseteq \alpha(S)$ for any set of states $S$, and $\alpha(S) \subseteq \alpha(T)$ for any set of states $T$ such that $S \subseteq T$. We apply abstraction after each application of the "one-step" operator post

$$
\operatorname{post}^{\#}(S)=\alpha(\operatorname{post}(S)),
$$

and then obtain the desired over-approximation of the reachable states

$$
\text { Reach }^{\#}=\operatorname{Ifp}\left(\text { post }^{\#},\left\{s_{I}\right\}\right) \text {. }
$$

The main challenge in applying the abstract interpretation framework amounts to choosing the abstraction function $\alpha$ that is precise enough and can be efficiently computed in practice.

Predicate abstraction is a prominent approach to automate the construction of $\alpha$ using automated theorem prover [12]. It requires a finite set of predicates Preds $=\left\{P_{1}, \ldots, P_{n}\right\}$, where each predicate $P_{i}$ represents a set of program states $P_{i} \subseteq \Sigma$. An over-approximation of the state is constructed from Preds. Automated refinement techniques are used to determine the set of predicates that define the abstraction function. 


\subsection{Algorithms}

We present our algorithm to combine the subsumer-first search strategy with the CEGAR framework. In each iteration of the abstract-check-refine loop, the method abstractCheck is called with queue $\mathcal{Q}$ initially containing only the start state. If no error state is reached and a fixpoint is reached, i.e. $\mathcal{Q}$ becomes empty, the program is declared SAFE. Otherwise if an error state is encountered, the abstract counterexample is checked whether it is a valid concrete counterexample. If yes, the counterexample is returned as a concrete counterexample presenting the violation of the safety property. Otherwise the spurious counterexample is refined by adding new predicates which refute its concrete existence and again a new iteration of abstractCheck is initiated with the newer set of predicates.

abstractCheck The abstractCheck algorithm in Figure 2 keeps expanding the states until either the queue becomes empty (line 1) or some error state is reached (line 5). It dequeues the first element $n$ in the queue $\mathcal{Q}$ (line 2). Then for all possible enabled transitions $\tau$, i.e. all transitions which can fire from $n$, the next state $m$ is computed using post\# (line 4 ). If $m$ is an error state, checkCounterexample method is called which verifies if the counterexample path from the root to state $m$ is spurious or not (line 6). SubsumedSubtree contains the list of nodes that are present in the subtree rooted at node $p$ which is subsumed by $m$. In line 9 , it is computed for all such $p$ and then their union is taken. The child pointer from the parent of $p, p_{p}$ is modified to now point to $m$ (lines 10-11). Also, in line 12 the child pointers of $p$ are accordingly moved to now point from $m$.

If the node $m$ is subsumed by some other node $p$ in the tree, then the node $m$ is not scheduled in $\mathcal{Q}$ (line 16). It should be noted that it can never be the case that $m$ subsumes some node in the tree and at the same time is subsumed by some other node in the tree. We maintain the invariant that no node present in the tree is subsumed by any other node present in the tree.

If no node subsumes $m$, we schedule $m$ in front of all the nodes that are present in both the SubsumedSubtree list and the queue $\mathcal{Q}$ (line 26). If no nodes present in the SubsumedSubtree are present in $\mathcal{Q}$ or the SubsumedSubtree is empty, $m$ is appended at the end of $\mathcal{Q}$ (line 24).

computePosition The function computePosition returns the index of the first node in the queue $\mathcal{Q}$ that is also present in the SubsumedSubtree. It traverses over all the nodes in $\mathcal{Q}$ from the beginning (line 1 ) and returns the least index $i$, such that $\mathcal{Q}(i) \in$ SubsumedSubtree (line 4). If no such $i$ is present, it returns -1 .

computeSubtree The function computeSubtree computes the nodes in the subtree rooted at the node SId using the abstract_child relation : $n \times \tau \rightarrow n$. Since there might be loops following the abstract_child pointers, computeSubtree keeps adding nodes to the subTree list until it converges (lines 2-6). The cycles 


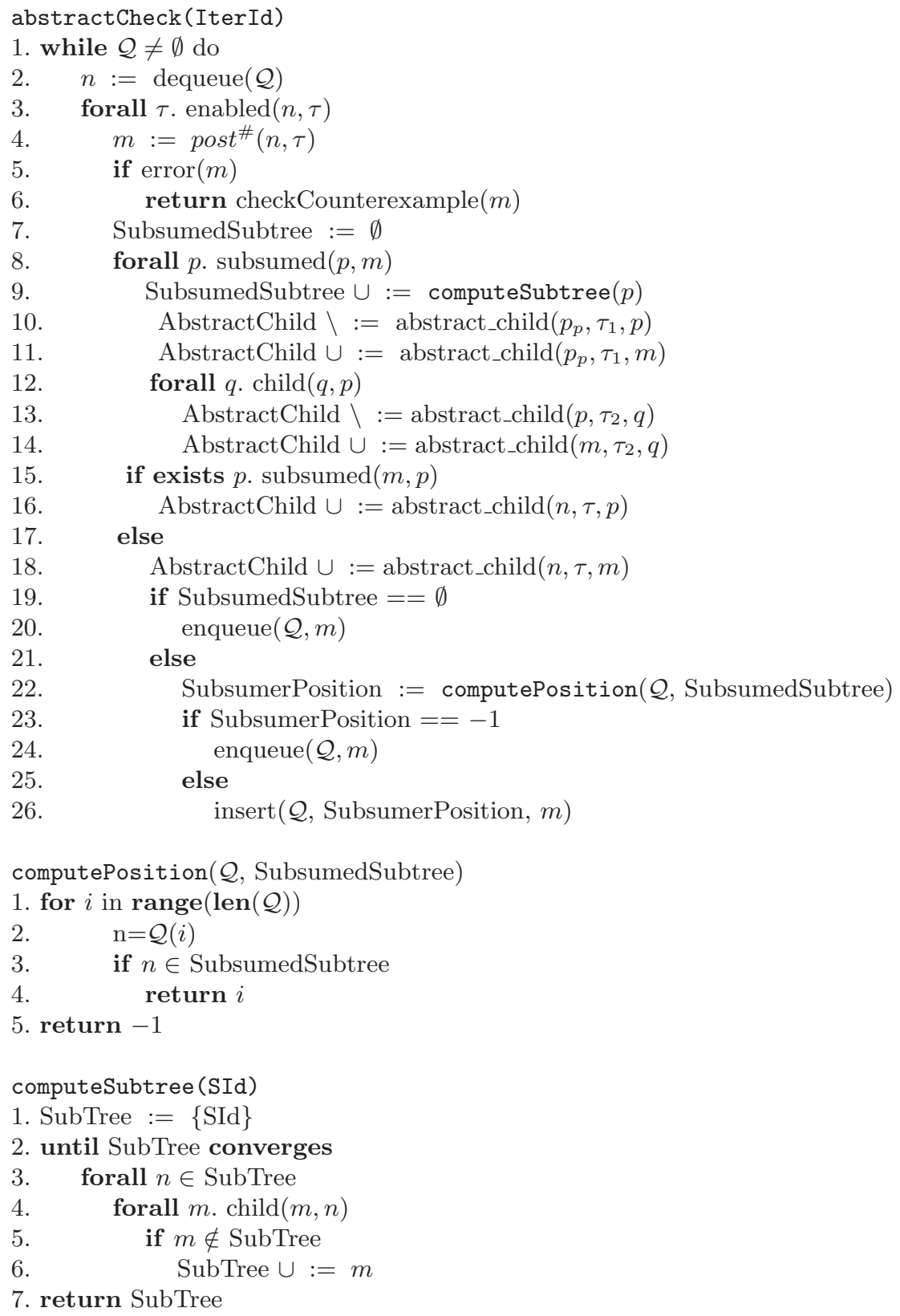

Fig. 2. Subsumer-first based algorithm for symbolic reachability analysis using predicate abstraction. 
(by following the child pointers) might be introduced while manipulating the child pointers when handling the subsumer and subsumed nodes.

\section{Experiments}

We present the results of the subsumer-first search heuristic and compare it with the breadth-first search strategy (both implemented in the ARMC model checker) on a set of benchmarks, some of which come from train control systems. We evaluated the heuristic on some of the most difficult benchmarks from the transportation domain. To get more coverage, we added some smaller benchmarks as well to the evaluation set. The results for computing the fixpoint given a fixed abstraction sufficient for verifying the safety property are presented in Table 1 and the results for the complete abstraction-refinement loop are presented in Table 2, see Appendix A. The first two rows in the tables present the performance of breadth-first search and subsumer-first search respectively. The third row presents the percentage decrease in the running time, entailment queries and the number of states. The experiments were run on a dual core 3.16 $\mathrm{GHz}$ Intel Pentium processor machine with $2 \mathrm{~GB}$ of RAM.

Table 1 presents the experiment results on the last iteration computation of ARMC given a sufficient set of predicates at the start of search to refute all spurious counterexamples and verify the safety property. This provides us a notion of reaching the fixpoint faster for a fixed abstraction. The last 3 columns of the table present the relative sizes of the benchmarks in terms of number of variables, transitions and locations in their control flow graphs. From the results, it is quite evident that subsumer-first strategy significantly outperforms the breadth-first search strategy consistently across all benchmarks. The subusmer-first strategy takes less time (mean decrease 31.8\%, median 33.3\%), produces fewer entailment queries (mean $35.8 \%$, median $42.1 \%$ ) and explores smaller number of states (mean $32.9 \%$, median 35.5\%). The subsumer-first strategy was on average 1.68 times faster, and the median speedup was 1.54 .

Table 2 presents the results for the complete abstraction-refinement loop starting with an empty initial abstraction. The results show that the subsumerfirst strategy mostly outperforms the breadth-first startegy in terms of running time(mean $31 \%$, median $46.9 \%$ ), number of entailment queries of theorem prover (mean 30.5\%, median 20.8\%) and the total number of states explored (mean $15.5 \%$, median $29.7 \%$ ). The subsumer-first strategy was 2.9 times faster on average (with median of 1.88) than the breadth-first strategy on these benchmarks. Since the subsumer-first approach refines thicker counterexamples, it usually finds more predicates but still on average reaches the fixpoint faster. In some cases, it might happen that while refining a shorter, thinner and local counterexample the breadth-first strategy might get lucky and the new predicates discovered may prune a large state space in the next iteration. But in general both from our experience from the experiments and as presented in [16], refining 
more counterexamples simultaneously provides a higher chance of discovering better predicates and faster fixpoint arrival.

Odometrys1ub and model-test19 seem to belong to those exception cases where the thicker counterexamples take some time to find the right set of predicates whereas the thinner counterexample finds a good set of predicates accidentally. But even then the subsumer-first strategy remarkably generated fewer entailment queries on the odometrys1ub benchmark.

\section{Discussion and future work}

In this paper we present a useful heuristic which addresses the issues of efficient counterexample discovery and faster convergence of reachability computation simultaneously. The subsumer-first heuristic can also be thought of as a combination of breadth-first search strategy for state exploration with depth-first exploration of subsumer nodes. It tries to get benefits of both approaches and produces short and thick counterexamples. Refining thicker counterexamples gives a better chance to get good predicates after refinement. These predicates can potentially rule out many spurious paths in later iterations. The optimality of the strategy can not be guaranteed as sometimes some lucky predicates can be discovered from other counterexamples which may prune the search space far more. However, in practice the subsumer-first strategy usually performs well.

This heuristic can easily be integrated with other heuristic state space exploration strategies to achieve even more savings, e.g in case of saturating the strongly connected components(SCC) first heuristic, the subsumer-first heuristic can be used inside a particular SCC during its saturation. We present in this paper one application of this heuristic in predicate abstraction based model checking. Its adaptation for integration with lazy abstraction and partial order reduction techniques would certainly be an interesting next step.

One interesting case occurs when a node $m$ subsumes a node $p$, we change the child pointer of parent of $p_{p}$ to $m$. But now if some node $n$ subsumes $p_{p}$, it may be the case that there are some states in the subtree of $m$ which are not reachable from $n$ but still we schedule $n$ before all of $m$ 's subtree. Using more fine-grained information about the transition system might help in predicting even better positions for scheduling the new nodes in the queue $\mathcal{Q}$.

\section{References}

1. T. Ball, R. Majumdar, T. D. Millstein, and S. K. Rajamani. Automatic predicate abstraction of C programs. In PLDI, pages 203-213. ACM, 2001.

2. T. Ball and S. K. Rajamani. Bebop: A symbolic model checker for boolean programs. In SPIN, pages 113-130, 2000.

3. F. Bourdoncle. Efficient chaotic iteration strategies with widenings. In Proceedings of the International Conference on Formal Methods in Programming and their Applications, pages 128-141. Springer-Verlag, 1993.

4. J. R. Burch, E. M. Clarke, K. L. McMillan, D. L. Dill, and L. J. Hwang. Symbolic model checking: $10^{20}$ states and beyond. Inf. Comput., 98(2):142-170, 1992. 
5. S. Chaki, E. M. Clarke, A. Groce, S. Jha, and H. Veith. Modular verification of software components in C. In ICSE, pages 385-395. IEEE Computer Society, 2003.

6. E. Clarke, O. Grumberg, S. Jha, Y. Lu, and H. Veith. Counterexample-guided abstraction refinement. In $C A V$, pages 154-169. Springer, 2000.

7. E. M. Clarke, O. Grumberg, and D. A. Peled. Model Checking. MIT Press, 1999.

8. P. Cousot and R. Cousot. Abstract interpretation: a unified lattice model for static analysis of programs by construction or approximation of fixpoints. In $P O P L$, pages 238-252. ACM, 1977.

9. K. Dräger, B. Finkbeiner, and A. Podelski. Directed model checking with distancepreserving abstractions. In SPIN, pages 19-34, 2006.

10. S. Edelkamp, S. Leue, and A. Lluch-Lafuente. Directed explicit-state model checking in the validation of communication protocols. STTT, 5(2-3):247-267, 2004.

11. S. Edelkamp, A. Lluch-Lafuente, and S. Leue. Directed explicit model checking with HSF-SPIN. In SPIN, pages 57-79, 2001.

12. S. Graf and H. Saidi. Construction of abstract state graphs with PVS. In $C A V$, pages 72-83. Springer, 1997.

13. A. Groce and W. Visser. Heuristic model checking for java programs. In SPIN, pages $242-245,2002$.

14. T. A. Henzinger, R. Jhala, R. Majumdar, and G. Sutre. Lazy abstraction. In POPL, pages 58-70. ACM, 2002.

15. S. Kupferschmid, K. Dräger, J. Hoffmann, B. Finkbeiner, H. Dierks, A. Podelski, and G. Behrmann. Uppaal/DMC- Abstraction-based heuristics for directed model checking. In TACAS, pages 679-682, 2007.

16. K. L. McMillan and N. Amla. Automatic abstraction without counterexamples. In $T A C A S$, pages $2-17,2003$.

17. D. Peled. All from one, one for all: on model checking using representatives. In $C A V$, pages 409-423, 1993.

18. A. Podelski and A. Rybalchenko. ARMC: The logical choice for software model checking with abstraction refinement. In $P A D L$, pages 245-259, 2007.

\section{A Evaluation results}

\begin{tabular}{|c|c|c|c|c|c|c|c|}
\hline Benchmark & time & \# queries & \# states & \# vars & $\#$ trans & \# locs & peedup \\
\hline $\begin{array}{l}\text { odometrys } 4 \mathrm{lb}^{1} \\
\text { odometrys } 4 \text { lb -sub-first }\end{array}$ & $\begin{array}{l}787 \mathrm{~m} \\
\mathbf{4 9 4 m} \\
37.2 \%\end{array}$ & $\begin{array}{l}18.3 \mathrm{M} \\
\mathbf{9 . 4 M} \\
48.6 \%\end{array}$ & $\begin{array}{l}11486 \\
\mathbf{6 2 0 7} \\
45.9 \%\end{array}$ & 15 & 3337 & 150 & 1.59 \\
\hline $\begin{array}{l}\text { odometryls2lb }{ }^{1} \\
\text { odometryls2lb -sub-first }\end{array}$ & $\begin{array}{l}227 \mathrm{~m} \\
\mathbf{1 0 4 m} \\
54.2 \%\end{array}$ & $\begin{array}{l}7.9 \mathrm{~m} \\
\mathbf{3 . 8 m} \\
51.9 \%\end{array}$ & $\begin{array}{c}8184 \\
\mathbf{3 8 8 6} \\
52.5 \%\end{array}$ & 16 & 6127 & 214 & 2.18 \\
\hline $\begin{array}{l}\text { odometryls1ub } \\
\text { odometryls1ub-sub-first }\end{array}$ & $\begin{array}{c}243 \mathrm{~m} \\
\mathbf{7 6 m} \\
68.7 \% \\
\end{array}$ & $\begin{array}{l}13.3 \mathrm{M} \\
4.5 \mathrm{M} \\
66.2 \%\end{array}$ & $\begin{array}{l}12762 \\
\mathbf{4 6 2 4} \\
63.8 \% \\
\end{array}$ & 16 & 6127 & 214 & 3.19 \\
\hline $\begin{array}{l}\text { odometrys1ub } \\
\text { odometrys1ub -sub-first }\end{array}$ & \begin{tabular}{|c|}
$34 \mathrm{~m}$ \\
$\mathbf{1 9 m}$ \\
$44.1 \%$
\end{tabular} & $\begin{array}{l}1.6 \mathrm{M} \\
\mathbf{0 . 7 M} \\
56.2 \%\end{array}$ & $\begin{array}{c}2073 \\
\mathbf{1 0 3 3} \\
50.2 \%\end{array}$ & 15 & 3337 & 150 & 1.79 \\
\hline
\end{tabular}

or RESOURCE-ERROR due to memory requirements 


\begin{tabular}{|c|c|c|c|c|c|c|c|}
\hline $\begin{array}{l}\text { timing } \\
\text { timing -sub-first }\end{array}$ & $\begin{array}{c}29 \mathrm{~m} \\
29 \mathrm{~m} \\
0 \%\end{array}$ & $\begin{array}{c}0.4 \mathrm{M} \\
\mathbf{0 . 3 9 M} \\
2.5 \%\end{array}$ & $\begin{array}{c}3425 \\
3378 \\
1.4 \%\end{array}$ & 47 & 99093 & 4954 & 1.0 \\
\hline $\begin{array}{l}\text { gasburner } \\
\text { gasburner -sub-first }\end{array}$ & $\begin{array}{l}17 \mathrm{~m} \\
\mathbf{9 m} \\
47 \%\end{array}$ & $\begin{array}{l}3.5 \mathrm{M} \\
1.7 \mathrm{M} \\
51.4 \%\end{array}$ & $\begin{array}{c}3309 \\
\mathbf{1 7 9 1} \\
45.9 \%\end{array}$ & 19 & 3124 & 152 & 1.89 \\
\hline $\begin{array}{l}\text { odometryls1lb } \\
\text { odometryls1lb -sub-first }\end{array}$ & \begin{tabular}{|c|}
$12 \mathrm{~m}$ \\
$\mathbf{4 m}$ \\
$66.7 \%$
\end{tabular} & $\begin{array}{c}0.8 \mathrm{M} \\
\mathbf{0 . 3 M} \\
62.5 \%\end{array}$ & $\begin{array}{c}1439 \\
\mathbf{6 3 2} \\
56 \%\end{array}$ & 16 & 6127 & 214 & 3.0 \\
\hline $\begin{array}{l}\text { rtalltcs } \\
\text { rtalltcs -sub-first }\end{array}$ & $\begin{array}{c}4 \mathrm{~m} \\
\mathbf{2 m} \\
50 \%\end{array}$ & $\begin{array}{c}2.5 \mathrm{M} \\
\mathbf{1 M} \\
60 \%\end{array}$ & $\begin{array}{c}1789 \\
796 \\
55.5 \%\end{array}$ & 20 & 18757 & 122 & 2.0 \\
\hline $\begin{array}{l}\text { odometrys1lb } \\
\text { odometrys1lb-sub-first }\end{array}$ & $\begin{array}{l}2 \mathrm{~m} \\
\mathbf{1 m} \\
50 \%\end{array}$ & $\begin{array}{c}0.2 \mathrm{M} \\
\mathbf{0 . 1 M} \\
50 \%\end{array}$ & $\begin{array}{c}681 \\
\mathbf{4 2 5} \\
37.6 \%\end{array}$ & 15 & 3337 & 150 & 2.0 \\
\hline $\begin{array}{l}\text { triple2 } \\
\text { triple2 -sub-first }\end{array}$ & $\begin{array}{l}2 \mathrm{~m} \\
\mathbf{2 m} \\
0 \%\end{array}$ & $\begin{array}{c}0.77 \mathrm{M} \\
\mathbf{0 . 7 0 M} \\
9 \%\end{array}$ & $\begin{array}{c}610 \\
\mathbf{5 2 0} \\
14.8 \%\end{array}$ & 3 & 8 & 3 & 1.0 \\
\hline $\begin{array}{l}\text { odometry } \\
\text { ododmetry -sub-first }\end{array}$ & $\begin{array}{c}1 \mathrm{~m} \\
\mathbf{4 0 s} \\
33.3 \%\end{array}$ & $\begin{array}{c}0.14 \mathrm{M} \\
\mathbf{0 . 0 9 M} \\
35.7 \%\end{array}$ & $\begin{array}{c}246 \\
\mathbf{1 9 3} \\
21.5 \%\end{array}$ & 15 & 437 & 28 & 1.5 \\
\hline $\begin{array}{l}\text { bakery3 } \\
\text { bakery3 -sub-first }\end{array}$ & $\begin{array}{l}11 \mathrm{~s} \\
\mathbf{1 0 s} \\
9 \%\end{array}$ & $\begin{array}{c}0.25 \mathrm{M} \\
\mathbf{0 . 1 9 M} \\
24 \%\end{array}$ & $\begin{array}{c}1311 \\
\mathbf{9 8 6} \\
24.8 \%\end{array}$ & 9 & 31 & 3 & 1.1 \\
\hline $\begin{array}{l}\text { model-test01 } \\
\text { model-test01 -sub-first }\end{array}$ & $\begin{array}{c}32 \mathrm{~s} \\
\mathbf{2 9 s} \\
9.4 \%\end{array}$ & $\begin{array}{c}0.18 \mathrm{M} \\
\mathbf{0 . 1 8 M} \\
0 \%\end{array}$ & $\begin{array}{c}1578 \\
\mathbf{1 5 6 5} \\
0.8 \%\end{array}$ & 16 & 110 & 36 & 1.1 \\
\hline $\begin{array}{l}\text { model-test07 } \\
\text { model-test07 -sub-first }\end{array}$ & $\begin{array}{c}39 \mathrm{~s} \\
37 \mathrm{~s} \\
5.1 \%\end{array}$ & $\begin{array}{c}0.25 \mathrm{M} \\
\mathbf{0 . 2 4 M} \\
4 \%\end{array}$ & $\begin{array}{c}1998 \\
\mathbf{1 9 0 2} \\
4.8 \%\end{array}$ & 16 & 124 & 40 & 1.05 \\
\hline $\begin{array}{l}\text { model-test13 } \\
\text { model-test13 -sub-first }\end{array}$ & $\begin{array}{l}\mathbf{2 m} \\
2 \mathrm{~m} \\
0 \%\end{array}$ & $\begin{array}{c}0.9 \mathrm{M} \\
\mathbf{0 . 7 5 M} \\
16.7 \%\end{array}$ & $\begin{array}{c}5766 \\
\mathbf{4 7 9 1} \\
16.9 \%\end{array}$ & 16 & 110 & 36 & 1.0 \\
\hline $\begin{array}{l}\text { model-test19 } \\
\text { model-test19 -sub-first }\end{array}$ & \begin{tabular}{|c}
$3 \mathrm{~m}$ \\
$\mathbf{2 m}$ \\
$33.3 \%$
\end{tabular} & $\begin{array}{c}0.9 \mathrm{M} \\
\mathbf{0 . 6 M} \\
33.3 \%\end{array}$ & $\begin{array}{c}5256 \\
\mathbf{3 4 9 9} \\
33.4 \%\end{array}$ & 16 & 124 & 40 & 1.5 \\
\hline
\end{tabular}

Table 1: Experiments with last iteration of ARMC with fixed set of predicates, fixpoint computation. 


\begin{tabular}{|c|c|c|c|c|c|c|}
\hline Benchmark & time & \# queries & \# iter & \# preds & \# states & speedup \\
\hline $\begin{array}{l}\text { odometry } \\
\text { odometry-sub-first }\end{array}$ & \begin{tabular}{|c|}
$109 \mathrm{~m}$ \\
$\mathbf{8 m}$ \\
$92.7 \%$
\end{tabular} & $\begin{array}{c}9.3 \mathrm{M} \\
\mathbf{1 . 6 M} \\
82.8 \%\end{array}$ & $\begin{array}{l}65 \\
37\end{array}$ & $\begin{array}{l}218 \\
153\end{array}$ & $\begin{array}{c}680 \\
\mathbf{2 9 5} \\
56.6 \%\end{array}$ & 13.6 \\
\hline $\begin{array}{l}\text { odometryls1lb } \\
\text { odometryls1lb -sub-first }\end{array}$ & \begin{tabular}{|c|}
$60 \mathrm{~m}$ \\
$\mathbf{2 9 m}$ \\
$51.7 \%$
\end{tabular} & $\begin{array}{c}7.1 \mathrm{M} \\
\mathbf{3 M} \\
57.7 \%\end{array}$ & $\begin{array}{l}32 \\
\mathbf{2 9}\end{array}$ & $\begin{array}{c}\mathbf{9 7} \\
102\end{array}$ & $\begin{array}{c}1439 \\
\mathbf{5 3 9} \\
62.5 \%\end{array}$ & 2.07 \\
\hline $\begin{array}{l}\text { triple2 } \\
\text { triple2 -sub-first }\end{array}$ & $\begin{array}{c}13 \mathrm{~m} \\
\mathbf{2 m} \\
84.6 \%\end{array}$ & $\begin{array}{c}6.5 \mathrm{M} \\
\mathbf{2 . 1 M} \\
67.7 \%\end{array}$ & $\begin{array}{l}65 \\
45\end{array}$ & $\begin{array}{l}254 \\
219\end{array}$ & $\begin{array}{c}519 \\
\mathbf{2 4 8} \\
52.2 \%\end{array}$ & 6.50 \\
\hline $\begin{array}{l}\text { odometrys1lb } \\
\text { odometrys1lb-sub-first }\end{array}$ & $\begin{array}{c}\mathrm{mm} \\
\mathbf{9 m} \\
0 \%\end{array}$ & $\begin{array}{c}1.1 \mathrm{M} \\
\mathbf{1 . 0 M} \\
9 \%\end{array}$ & $\begin{array}{l}\mathbf{2 0} \\
22\end{array}$ & $\begin{array}{l}72 \\
83\end{array}$ & $\begin{array}{c}681 \\
\mathbf{3 4 5} \\
49.3 \% \\
\end{array}$ & 1.0 \\
\hline $\begin{array}{l}\text { odometrys1ub } \\
\text { odoemtrys1ub-sub-first }\end{array}$ & \begin{tabular}{|c|}
$\mathbf{1 9 5 m}$ \\
$329 \mathrm{~m}$ \\
$-68.7 \%$ \\
\end{tabular} & $\begin{array}{c}14.4 \mathrm{M} \\
\mathbf{1 1 . 4 M} \\
20.8 \%\end{array}$ & $\begin{array}{l}37 \\
33\end{array}$ & $\begin{array}{l}\mathbf{1 5 7} \\
257\end{array}$ & $\begin{array}{c}\mathbf{2 0 7 3} \\
2379 \\
-14.8 \% \\
\end{array}$ & 0.59 \\
\hline $\begin{array}{l}\text { gasburner } \\
\text { gasburner -sub-first }\end{array}$ & \begin{tabular}{|c|}
$175 \mathrm{~m}$ \\
$\mathbf{9 3 m}$ \\
$46.9 \%$ \\
\end{tabular} & $\begin{array}{c}48.9 \mathrm{M} \\
\mathbf{1 7 . 3 M} \\
64.6 \%\end{array}$ & $\begin{array}{l}64 \\
61\end{array}$ & $\begin{array}{l}\mathbf{1 9 8} \\
220\end{array}$ & $\begin{array}{c}3309 \\
\mathbf{1 6 0 4} \\
51.5 \%\end{array}$ & 1.88 \\
\hline $\begin{array}{l}\text { timing } \\
\text { timing -sub-first }\end{array}$ & $\begin{array}{l}51 \mathrm{~m} \\
\mathbf{4 9 m} \\
3.9 \%\end{array}$ & $\begin{array}{c}1 \mathrm{M} \\
1 \mathrm{M} \\
0 \%\end{array}$ & $\begin{array}{l}14 \\
14\end{array}$ & $\begin{array}{l}14 \\
14\end{array}$ & $\begin{array}{c}3425 \\
3378 \\
1.4 \%\end{array}$ & 1.04 \\
\hline $\begin{array}{l}\text { rtalltcs } \\
\text { rtalltcs -sub-first }\end{array}$ & $\begin{array}{l}38 \mathrm{~m} \\
\mathbf{3 7 m} \\
2.6 \%\end{array}$ & $\begin{array}{c}27 \mathrm{M} \\
\mathbf{2 5 . 3 M} \\
6.3 \%\end{array}$ & $\begin{array}{l}30 \\
40\end{array}$ & $\begin{array}{l}\mathbf{5 6} \\
74\end{array}$ & $\begin{array}{c}1789 \\
\mathbf{1 2 5 8} \\
29.7 \%\end{array}$ & 1.03 \\
\hline $\begin{array}{l}\text { bakery3 } \\
\text { bakery3 -sub-first }\end{array}$ & $\begin{array}{c}2 \mathrm{~m} \\
\mathbf{3 2 s} \\
73.3 \%\end{array}$ & $\begin{array}{c}2.6 \mathrm{M} \\
\mathbf{0 . 9 M} \\
65.4 \%\end{array}$ & $\begin{array}{l}\mathbf{3 4} \\
36\end{array}$ & $\begin{array}{l}67 \\
\mathbf{5 8}\end{array}$ & $\begin{array}{c}1419 \\
\mathbf{8 8 5} \\
37.6 \%\end{array}$ & 3.75 \\
\hline $\begin{array}{l}\text { model-test01 } \\
\text { model-test01 -sub-first }\end{array}$ & $\begin{array}{c}4 \mathrm{~m} \\
\mathbf{2 m} \\
50 \%\end{array}$ & $\begin{array}{c}1.7 \mathrm{M} \\
\mathbf{1 . 5 M} \\
11.8 \%\end{array}$ & $\begin{array}{l}58 \\
\mathbf{5 4}\end{array}$ & $\begin{array}{l}115 \\
100\end{array}$ & $\begin{array}{c}\mathbf{1 2 0 7} \\
1565 \\
-29.7 \%\end{array}$ & 2.0 \\
\hline $\begin{array}{l}\text { model-test07 } \\
\text { model-test07 -sub-first }\end{array}$ & $\begin{array}{c}5 \mathrm{~m} \\
\mathbf{3 m} \\
40 \%\end{array}$ & $\begin{array}{c}2.4 \mathrm{M} \\
\mathbf{2 . 2 M} \\
8.3 \%\end{array}$ & $\begin{array}{l}58 \\
\mathbf{5 6}\end{array}$ & $\begin{array}{l}115 \\
\mathbf{1 0 4}\end{array}$ & $\begin{array}{c}\mathbf{1 3 7 2} \\
1902 \\
-38.6 \%\end{array}$ & 1.67 \\
\hline $\begin{array}{l}\text { model-test13 } \\
\text { model-test13 -sub-first }\end{array}$ & $\begin{array}{c}17 \mathrm{~m} \\
\mathbf{9 m} \\
47 \% \\
\end{array}$ & $\begin{array}{c}6.6 \mathrm{M} \\
\mathbf{5 . 2 M} \\
21.2 \%\end{array}$ & $\begin{array}{l}63 \\
61\end{array}$ & $\begin{array}{l}140 \\
\mathbf{1 3 6}\end{array}$ & $\begin{array}{c}\mathbf{4 7 0 8} \\
4791 \\
-1.8 \% \\
\end{array}$ & 1.89 \\
\hline $\begin{array}{l}\text { model-test19 } \\
\text { model-test19 -sub-first }\end{array}$ & $\begin{array}{c}\mathbf{1 9 m} \\
23 \mathrm{~m} \\
-21 \%\end{array}$ & $\begin{array}{c}\text { 7.7M } \\
9.2 \mathrm{M} \\
-19.5 \%\end{array}$ & $\begin{array}{l}62 \\
\mathbf{5 9}\end{array}$ & $\begin{array}{l}137 \\
\mathbf{1 3 5}\end{array}$ & $\begin{array}{c}\mathbf{5 2 5 6} \\
8135 \\
-54.8 \%\end{array}$ & 0.83 \\
\hline
\end{tabular}

Table 2. Experiments with full ARMC abstraction-refinement iterations 